\title{
Prescription for an ailing pharmaceutical industry
}

\author{
Arnold L. Demain
}

A major change has occurred in the pharmaceutical industry in the past several years. This has been caused in part by the financial community's expectation that a certain number of blockbuster drugs per year are necessary for company profitability. As a result, we have witnessed an extensive series of mergers among the giants of the industry as well as their acquisitions of smaller biopharmaceutical companies. This has unfortunately not solved the financial problem: the commercial output of the combined companies has not even equaled the total number of products commercialized by the organizations existing before merger mania took over.

There are several reasons for this inefficiency. First, the number of competing discovery groups, each with its own concepts, methodologies, and ingenuity, has decreased. The newly merged companies tend to be less innovative than the original organizations.

Second, there is a perceived need to feed the discovery machine with more and more candidate compounds. High-throughput screening, which began in the early 1990s, can assay as many as 100,000 entities in one day. It has accomplished miniaturization and speed, but has neither provided the numbers of highquality leads anticipated nor even increased the rate at which such leads are found.

This worship of the numbers game has virtually eliminated the most unique source of chemical diversity, natural products, from the playing field in favor of combinatorial chemistry. It is not that Nature does not provide millions of new compounds, but that it is more difficult and takes more brainpower to harness and analyze this great feast of undiscovered chemistry by high-throughput screening. Combinatorial chemistry yields mainly minor modifications of present-day drugs, and without new scaffolds on which to build, it is not up to the task at hand.

A third reason relates to the promise of genomics. Although comparative genomics can disclose new targets for antimicrobials and other types of drugs, the number of such potential targets is so large that it requires tremendous investments of time and money to set up all the screens necessary to exploit this resource. This can only be handled by a

Arnold L. Demain is fellow of the Charles A. Dana Research Institute for Scientists

Emeriti, 330 Hall of Science, Drew University, Madison, NJ07940(ademain@drew.edu). high-throughput screening methodology, which demands libraries of millions of chemical entities. Although such targets would be excellent for screening natural products, the industry has failed to exploit this opportunity and has opted to save funds by eliminating natural product departments or decreasing their relevance in the hunt for new drugs.

Finally, an ever-increasing number of synthetic drugs have been recalled by the US Food and Drug Administration (Rockville, MD) because of toxicities and deaths occurring in patients after initial approval.

During the 3 billion years that bacteria have inhabited the earth, Nature has conducted its own type of combinatorial chemistry in a much more exotic way than chemists, leading to the creation of hundreds of thousands of novel secondary metabolites. These natural products, with structures more spatially complex than those of synthetic chemicals, have been overwhelmingly useful to our society.

Microbial and plant secondary metabolites doubled our life span during the 20th century. They have reduced pain and suffering, and revolutionized medicine. Over half of approved drugs are either natural products or related to them, even excluding biologicals, such as vaccines and monoclonal antibodies. It is most unfortunate that the pharmaceutical industry has downgraded natural products just at the time that new assays are available and major improvements have been made in detection, characterization, and purification of small molecules. With the advent of combinatorial biosynthesis, thousands of new derivatives can now be made by a biological technique complementary to combinatorial chemistry. Furthermore, only a minor proportion $(-1-10 \%)$ of bacteria and fungi have thus far been examined for secondary metabolite production. New methods are being developed to cultivate the so-called "unculturable" microbes from the soil and sea.

The selective action exerted on pathogenic bacteria and fungi by microbial secondary metabolites ushered in the antibiotic era, and for over 50 years, we have benefited from this remarkable property of "wonder drugs". The market for such compounds is over $\$ 30$ billion per year. Microbiologists know that antimicrobial technology alone will not permanently win the war against infectious microorganisms because of the development of resistance in pathogenic microbes. We will have to be satisfied merely to stay one step ahead of the pathogens for a long time to come; thus, the search for new antibiotics must not be stopped.

New entities are continually needed as a result of the development of resistant pathogens, the existence of naturally resistant bacteria (e.g.) Pseudomonas aeruginosa, Stenotrophomonas maltophilia, many enterococci, Burkholderia cepacia and Acinetobacter baumanni), and the toxicity of some of the current compounds. Another problem is the increased incidence of infection by organisms that are not normally virulent but do infect immunocompromised patients. Fungal infections doubled from the 1980s to the 1990s. Candidiasis, cryptococcosis, and aspergillosis are on the rise, and the rate of treatment failure in aspergillosis exceeds $60 \%$. Fungal infections, usually by Candida and Aspergillus species, often occur after lung, kidney, heart, and liver transplant operations. Pulmonary aspergillosis is the main factor in the death of recipients of bone marrow transplants, and Pneumocystis carinii is the primary cause of death in patients with AIDS from Europe and North America. Current treatments include the synthetic azoles (such as fluconazole and flucytosine) or the natural polyene amphotericin B. However, their use is limited by the development of resistance to the azoles and by the toxicity of amphotericin B.

In the past 20 years, there has been a major change in the discovery and application of secondary metabolites, characterized by a broadening of the scope of the search. No longer are microbial sources looked upon solely as providing potential solutions for microbial diseases. This change in screening philosophy has been followed by ingenious applications of molecular biology to detect activities of compounds from microbes and plants for non-antibiotic applications. These include the cholesterol-lowering statins (e.g., lovastatin and pravastatin), anticancer agents (e.g., the microtubule stabilizer paclitaxel), immunosuppressants (e.g., cyclosporin A, tacrolimus, sirolimus, and mycophenolic acid), antiparasitic agents (e.g., avermectins and the polyethers), bioherbicides (e.g, bialaphos), and bioinsecticides (e.g., the spinosins).

It is clear that the future success of the drug industry depends on the combination of complementary technologies, such as natural product discovery, high-throughput screening, genomics, proteomics, and combinatorial chemistry. Elimination of natural products from the mix is a formula for failure. 